\title{
MicroRNA-361-5p slows down gliomas development through regulating UBR5 to elevate ATMIN protein expression
}

\author{
Jiaoying Jia ${ }^{1}$, Zhu Ouyang ${ }^{1}$, Ming Wang ${ }^{1}$, Wenjia Ma ${ }^{1}$, Min Liu ${ }^{1}$, Mingming Zhang ${ }^{1,2}$ and Mengqiang Yu ${ }^{1,2}{ }^{凶}$ \\ (c) The Author(s) 2021
}

MicroRNA (miR)-361-5p has been studied to suppress gliomas development. Based on that, an insight into the regulatory mechanism of miR-361-5p in gliomas was supplemented from ubiquitin protein ligase E3 component N-recognin 5 (UBR5)mediated ubiquitination of ataxia-telangiectasia mutated interactor (ATMIN). miR-361-5p, ATMIN, and UBR5 levels were clinically analyzed in gliomas tissues, which were further validated in gliomas cell lines. Loss/gain-of-function method was applied to determine the roles of miR-361-5p and UBR5 in gliomas, as to cell viability, migration, invasion, colony formation ability, and apoptosis in vitro and tumorigenesis in vivo. The relationship between miR-361-5p and UBR5 was verified and the interaction between UBR5 and ATMIN was explored. It was detected that reduced miR-361-5p and ATMIN and enhanced UBR5 levels showed in gliomas. Elevating miR-361-5p was repressive in gliomas progression. UBR5 was directly targeted by miR-361-5p. UBR5 can ubiquitinate ATMIN. miR-361-5p suppressed gliomas by regulating UBR5-mediated ubiquitination of ATMIN. Downregulating UBR5 impeded gliomas tumor growth in vivo. Upregulating miR-361-5p targets UBR5 to promote ATMIN protein expression, thus to recline the malignant phenotype of gliomas cells.

Cell Death and Disease (2021)12:746; https://doi.org/10.1038/s41419-021-04010-1

\section{INTRODUCTION}

Gliomas form a heterogeneous group of central nervous system tumors [1] and are classified based on WHO Classification of CNS tumors published in 2016 [2]. Genetic risk factors, ionizing radiation, cellular phones, allergies and lifestyle, and diet are suggested as the risk factors for the occurrence and development of gliomas [3]. At present, variable therapies have been developing to treat the disease, and oncolytic virus therapy, stem cell therapy, immunotherapy, and electric field therapy are now on the market to improve the treatment prospects of recurrent high-grade gliomas [4]. The unsatisfactory outcome after treatment limits the survival of gliomas $\mathrm{p}$ authors are co-corresponding authoatients, thus an improved therapeutic approach is at wanting.

Effective delivery of miRNA alone or as part of a cytotoxic drug composition has been promising to improve clinical treatment of gliomas [5]. miR-361-5p is emerged as a regulatory actor in gliomas aerobic glycolysis, cell growth, and apoptosis [6]. Meanwhile, miR-361-5p stands for a repressor for matrix metalloproteinase (MMP)-2 transcription in gliomas migration and invasion [7]. Of significance, the process of epithelial-to-mesenchymal transition (EMT) in gliomas can be delayed by miR-361-5p targeting Twist1 [8]. Interestingly, a novel regulatory pattern of miR-361-5p in glioblastoma (GBM) implies its involvement in cancer progression and therapeutic potential [9].

Ubiquitin-proteasome system is a well-designed system that controls proteins by realizing a dynamic balance between ubiquitination and deubiquitination to control oncoproteins, tumor suppressors, and cell signaling pathways [10]. Ubiquitin protein ligase E3 component N-recognin 5 (UBR5) is a ubiquitin E3 ligase that mediates the degradation and ubiquitination of proteins involved in cancers $[11,12]$. It has been testified that UBR5 interacts with ataxia-telangiectasia mutated (ATM) interactor (ATMIN) and enhances ubiquitination of ATMIN at lysine 238, thus weakening ATMIN interaction with ATM [13]. The oncogenic activities of ATMIN have been discussed in human cancer, such as head and neck cancer [14]. In GBM, inactivating ATMIN/ATM pathway ascribes to the inhibited cancer aggravation [15]. Though miR-361-5p, UBR5, and ATMIN have been mentioned in gliomas-related mechanisms, whether they could cooperate to modulate gliomas cell fate still lacks further exploration. Thus, this research was initiated to decipher miR-361-5p/UBR5/ATMIN axis in gliomas.

\section{MATERIALS AND METHODS Ethics statement}

Experimental approval was gained from the ethics committee of The Second Xiangya Hospital of Central South University. A signed consent was provided by every participant. Animals were treated as the care and use of laboratory animals, and were approved by the animal research ethics committee of The Second Xiangya Hospital of Central South University.

\section{Clinical samples}

Gliomas tissue samples were collected from 98 patients with gliomas who received general surgery in The Second Xiangya Hospital of Central South

\footnotetext{
${ }^{1}$ Department of Neurosurgery, The Second Xiangya Hospital of Central South University, Changsha, Hunan 410011, China. ${ }^{2}$ These authors are co-corresponding authors. 凶email: zhangmm@csu.edu.cn; yumengqiang@csu.edu.cn

Edited by B. Joseph
}

Received: 1 December 2020 Revised: 21 April 2021 Accepted: 13 May 2021

Published online: 28 July 2021 
Table 1. Primer sequences.

\begin{tabular}{|c|c|c|}
\hline Primer sequences & Forward $\left(5^{\prime} \rightarrow 3^{\prime}\right)$ & Reverse $\left(5^{\prime} \rightarrow 3^{\prime}\right)$ \\
\hline miR-361-5p & AGGGGTACGTCGTATCCAGT & GTATCCAGTGCGTGTCGTGG \\
\hline UBR5 & ACGAGAAGGAAAGCACCATG & CTTCTCAGAAACTTCTCGTAAC \\
\hline ATMIN & AACAGCACTGCAGTCTCACA & CTGGTCTAGGGATTGGTTGGT \\
\hline Bax & CCAGCTCTGAGCAGATCATG & TGCTGGCAAAGTAGAAAAGG \\
\hline $\mathrm{Bcl}-2$ & GACTTCGCCGAGATGTCCAG & CAGGTGCCGGTTCAGGTACT \\
\hline U6 & GCACATTCTCCCCAGTTATGA & TCACAAATTTGCATGTCATCCT \\
\hline
\end{tabular}

miR-361-5p microRNA-361-5p, UBR5 Ubiquitin protein ligase E3 component N-recognin 5, ATMIN ataxia telangiectasia mutated interactor, Bax Bcl-2-associated $\mathrm{X}, \mathrm{BCl}-2 \mathrm{~B}$ cell lymphoma 2; GAPDH, glyceraldehyde-3-phosphate dehydrogenaseglyceraldehyde-3-phosphate dehydrogenase.

University, including 52 males and 46 females, 24-69 years old, with an average age of ( $46.5 \pm 7.52)$ years old. Among them, 41 cases were in WHO grade 2,32 cases in WHO grade 3 , and 25 cases in WHO grade 4 . Patients were confirmed with gliomas by pathological examination and the clinical data were complete. Any gliomas treatment was not performed in the past 3 months. Healthy brain tissues $(n=20)$ from the donors died of accidents were used as controls [16].

\section{Cell culture}

GBM cell line U251 (XY-XB-1030, Shanghai XYBIO Biotechnology, Shanghai, China), the other GBM cell lines U87, LN229, and A172 (ATCC, VA, USA) and normal human astrocytes NHA (DC790, Shanghai Ze Ye Biotechnology Co., Ltd., Shanghai, China) were cultured in Dulbecco's Modified Eagle Medium (DMEM, Invitrogen, CA, USA) supplemented with $10 \%$ fetal bovine serum (FBS, Hyclone, UT, USA) [17].

\section{Cell transfection}

miR-361-5p mimic and inhibitor sequence and its corresponding negative control (NC) sequence dsRNA oligonucleotides were available from Ribobio (Guangzhou, China). U87 and U251 cells growing to 50\% confluence were transfected with corresponding dsRNA oligonucleotides $(2 \mu \mathrm{g})$ through $10 \mu \mathrm{L}$ X-tremeGENE siRNA Transfection Reagent (Hoffmann-La Roche Ltd., Basel, Switzerland). The final concentration in transfection was $50 \mathrm{nM}$ [7]. The siRNA Smartpool and RISCfree control siRNA against human and mouse UBR5 (Thermo Fisher Scientific, Massachusetts, USA) were transfected into U87 and U251 cells through Dharmafect 1 (Invitrogen) [13].

\section{Reverse transcription-quantitative polymerase chain reaction (RT-qPCR)}

Total RNA was extracted from cells and frozen gliomas tissues with Trizol reagent (Invitrogen, Thermo Fisher). Gene-specific primers were used to synthesize miR-361-5p cDNA from total RNA. RT-qPCR determined gene expression via SYBR-Green PCR Master Mix kit. Gene mRNA levels were calculated by $2^{-\triangle \Delta C T}$ method [18], and normalized to $U 6$ and glyceraldehyde3-phosphate dehydrogenase (GAPDH) [19]. Table 1 showed the primers.

\section{Western blot assay}

Total protein lysate was prepared by treating cells with radioimmunoprecipitation assay (RIPA) buffer (Beyotime, Shanghai, China) and centrifugation at $17,000 \times g$. The protein concentration was determined with a bicinchoninic acid kit (Beyotime, Nantong, China). The total protein lysate was denatured by $10 \%$ sodium dodecyl sulfatepolyacrylamide gel electrophoresis (SDS-PAGE), transferred to polyvinylidene fluoride membranes, and sealed with $5 \%$ skimmed milk. Anti-human UBR5 antibody (1:1000) was obtained from immunizing rabbits with human UBR5 peptide (KWSEPYRNAQNPS) [20]. The primary antibodies against UBR5, MMP-2 (ab37150, 1:1000; Abcam), ATMIN (ab3271, 1:5000; Millipore Sigma), Bax (ab32503, 1:1000, Abcam), Bcl-2 (ab182858,1:2000; Abcam) and GAPDH (ab9482, 1:1000; Abcam) were probed with the membrane. Subsequently, the membrane was further probed with horseradish peroxidase-conjugated secondary antibody (1:2000; Cell Signaling Technology, Boston, USA), developed using an enhanced chemiluminescence kit (Beyotime), and visualized by Chemlmager5500 V2.03 software (Alpha Innotech, San Lean, USA). Using GAPDH as an internal control, the integrated density value of the protein band was quantified [20].

\section{Transwell assay}

Cell invasion assay was performed with a 24-well Transwell (Corning, USA). The Transwell chamber was coated with BD Matrigel matrix (Corning), and U87 and U251 cells $\left(3 \times 10^{4}\right)$ were seeded on the BD Matrigel in the upper chamber with serum-free medium. The lower chamber was filled with complete medium containing $10 \%$ FBS. Cell invasion was observed in six fields under a fluorescence microscope.

In the Transwell migration test, U87 and U251 cells $\left(3 \times 10^{4}\right)$ were suspended in serum-free medium and placed in the upper chamber without BD Matrigel matrix. The lower chamber was supplemented with serum-free medium. After $36 \mathrm{~h}$, cells were fixed with $4 \%$ paraformaldehyde and stained with $0.5 \%$ crystal violet [21].

\section{Colony formation assay}

Cell suspension was cultured in 10\% FBS-Roswell Park Memorial Institute1640 for $10 \mathrm{~d}$ in a 6 -well plate at 400 cells/well. Then, the colonies were fixed with anhydrous methanol and stained with $0.1 \%$ crystal violet. The colonies larger than $2 \mathrm{~mm}$ were counted [22].

\section{Flow cytometry}

Annexin 8-fluorescein isothiocyanate (FITC)/propidium iodide (PI) method was adopted to detect cell apoptosis. Cells were incubated 6-well plates for $48 \mathrm{~h}$, washed with $1 \times$ binding buffer $(185 \mathrm{~mL})$, and incubated with $5 \mu \mathrm{L}$ AnnexinV FITC and $5 \mu \mathrm{L}$ PI. Cell apoptosis was detected by FACSCalibur flow cytometer and Cell Quest Pro software (Thermo Fisher). The data were analyzed by FlowJo 7.6 software [23].

\section{3-(4,5-dimethylthiazol-2-yl)-2,5-diphenyltetrazolium bromide (MTT) assay}

After transfection of $24 \mathrm{~h}$, cells were trypsinized and grew in 96-well culture plates at 3000 cells/well. Cultured for $0,24,48$, and $72 \mathrm{~h}$, cells were combined with $\mathrm{MTT}$ solution $(10 \mu \mathrm{L}, 5 \mathrm{mg} / \mathrm{mL}$; Sigma-Aldrich, St. Louis, Missouri, USA) for another 4-h incubation, and reacted with dimethyl sulfoxide $(150 \mu \mathrm{L})$. The absorbance was measured at $490 \mathrm{~nm}$ with a SpectraMax M3 microplate reader (Molecular Equipment Corporation, LLC, Sunnyvale, CA, USA). Each group set five replicates [24].

\section{Immunocoprecipitation}

The cell lysate was successively incubated with anti-FLAG M2-Sepharose affinity gel (Sigma) for $18 \mathrm{~h}$, and specific antibody for $1 \mathrm{~h}$, protein $\mathrm{A} / \mathrm{G}$ agarose gel (Santa Cruz Biotechnology) for $18 \mathrm{~h}$. The pellets were slowly centrifuged, washed four times in lysis buffer or RIPA buffer, treated with ubiquitination or SDS-PAGE, and incubated with specific antibodies [20].

The constructed HA-ubiquitin plasmid and the indicated plasmid were transfected into gliomas cells. Cells were reacted with $25 \mu \mathrm{M}$ proteasome inhibitor MG132 (CalBiochem, La Jolla, California) for $5 \mathrm{~h}$. Cells at $48 \mathrm{~h}$ post transfection were lysed in RIPA buffer, immunoprecipitated with anti-ATMIN protein antibodies. The bound protein was eluted with Laemmli sample buffer and analyzed by western blot using anti-HA antibody. With cell membrane removal, proteins were re-detected with anti-ATMIN [20]. 

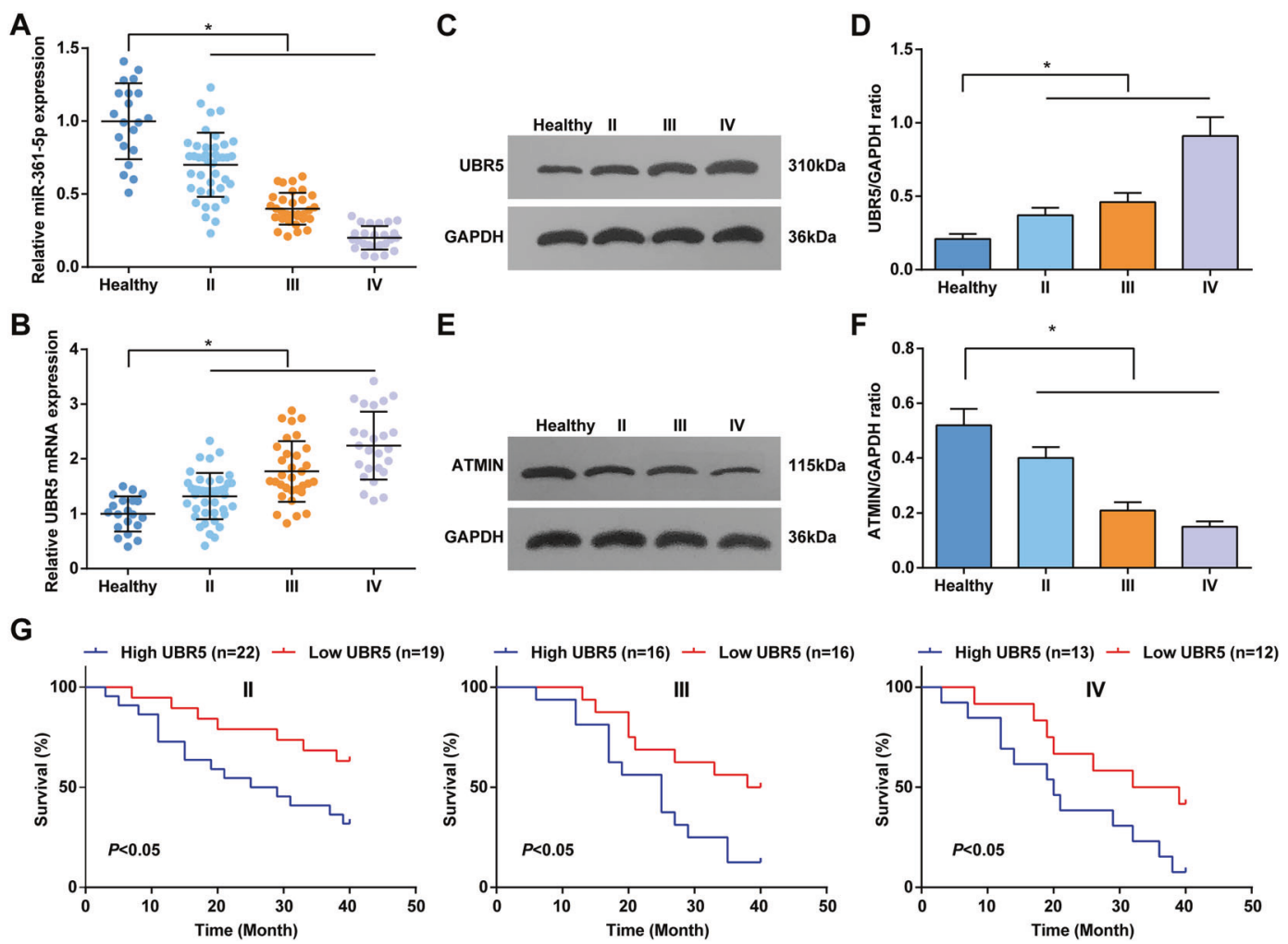

Fig. 1 miR-361-5p and ATMIN are upregulated and UBR5 is downregulated in gliomas. A RT-qPCR tested miR-361-5p expression in brain tissues of healthy controls and patients with gliomas at different stages. B RT-qPCR tested UBR5 mRNA expression of UBR5 in brain tissues of healthy controls and patients with gliomas at different stages. C, D Western blot tested UBR5 protein expression in brain tissues of healthy controls and patients with gliomas at different stages. E, F Western blot tested ATMIN protein expression in brain tissues of healthy controls and patients with gliomas at different stages. G Kaplan-Meier survival curve analyzed the relationship between UBR5 expression and the prognosis of patients with gliomas at different stages, univariate analysis was performed by Log-rank test; the data were all measurement data, in the form of mean \pm standard deviation; ${ }^{*} P<0.05$ indicated the relationship between patients with gliomas at different stages.

\section{Dual-luciferase reporter gene assay}

U87 and U251 cells were co-transfected with UBR5-wild type (Wt) or UBR5mutant type (Mut) and miR-361-5p mimic or mimic NC. After $24 \mathrm{~h}$, cells were analyzed in a dual-luciferase reporter analysis system (Promega, Madison, WI, USA). Luciferase activity = firefly luciferase intensity/Renilla luciferase intensity [25].

\section{Tumor xenografts in nude mice}

U87 cells $\left(5 \times 10^{6}\right.$ cells in $100 \mu \mathrm{L}$ PBS) transfected with si-NC and si-UBR5 were subcutaneously injected into the left axilla of male BALB/c nude mice (4 weeks old, Cell bank of Chinese Academy of Sciences, Shanghai, China). Each group was set with 8 mice. The tumor volume was measured every $3 \mathrm{~d}$ (volume $=1 / 2 \times$ length $\times$ width $^{2}$ ) [26]. The tumor tissues were paraffin-embedded, sliced to $4-\mu \mathrm{m}$ sections, and stained with anti-Ki-67 (DAKO) for immunohistochemical staining and TUNEL staining. The image was captured with AxioVision Rel.4.6 (Carl Zeiss). The cell proliferation index was quantified by counting the proportion of Ki-67-positive cells. The apoptosis index was calculated as the percentage of TUNEL-positive cells [27].

\section{Statistical analysis}

SPSS21.0 software (IBM, NY, USA) was employed to data analysis. All data conformed to normal distribution and homogeneous variance. Measurement data were expressed as mean \pm standard deviation. Two sets of data were compared by independent sample $t$ test, and data among multiple groups were compared by one-way analysis of variance (ANOVA), and Tukey's post hoc test. Survival rate of patients was calculated by Kaplan-Meier method, univariate analysis was performed by Log-rank test, and correlation analysis was implemented with Pearson test. $P<0.05$ was recorded as statistical difference.

\section{RESULTS}

miR-361-5p and ATMIN are downregulated and UBR5 is upregulated in gliomas

miR-361-5p could target Twist 1 to inhibit EMT of gliomas cells and presents a downregulated level in gliomas [8]. Detected by RTqPCR, lowly expressed miR-361-5p showed in gliomas tissues (Fig. $1 A)$. In addition, miR-361-5p level was lower in stage III tissues and the lowest in stage IV tissues, further indicating that miR-361-5p level was decreased as the tumor progressed.

Western blot detected ATMIN protein expression in patients, and the results suggested that ATMIN protein expression in glioma tissues was lower than that in normal tissues (Fig. 1E, F).

RT-qPCR and western blot measured UBR5 level and found that UBR5 was upregulated in gliomas tissues, especially in patients with stage IV gliomas (Fig. 1B-D). Thus, UBR5 level was considered to positively correlate with the WHO grade and gliomas progression. Taking the median of UBR5 mRNA expression as the cut-off value, patients with gliomas were divided into UBR5 low expression group and UBR5 high expression group and analyzed by Kaplan-Meier. The results revealed that survival rate of gliomas patients in different tumor grades with low UBR5 expression was higher (Fig. 1G) [28].

\section{Reduced miR-361-5p and ATIMN and enhanced UBR5 levels} show in gliomas cells

To further verify the expression tendency of miR-361-5p, ATIMN, and UBR5 in gliomas, their levels in gliomas cell lines (U87, U251, LN229, and A172) and NHA were measured. As tested, miR-361-5p 

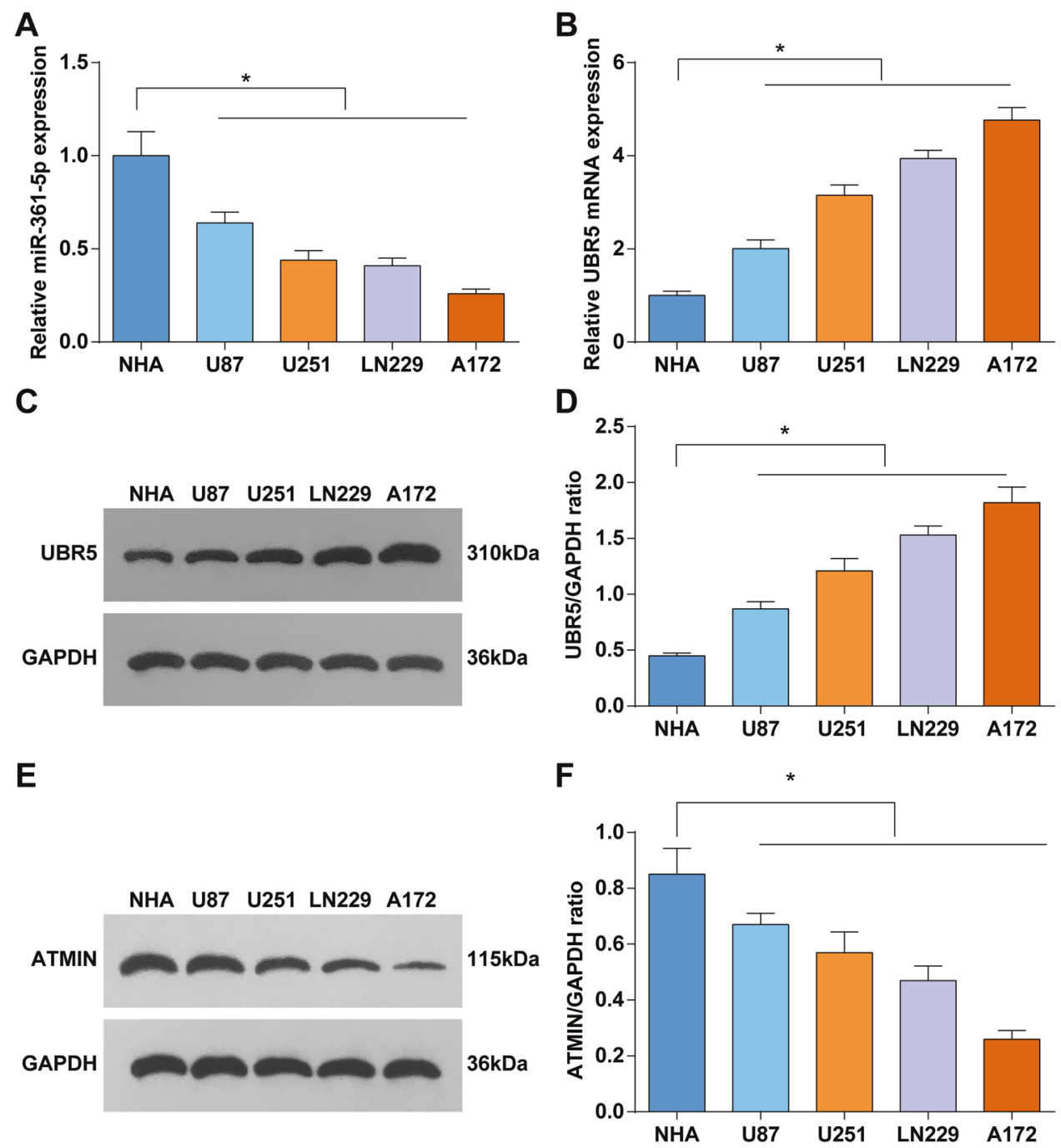

Fig. 2 Reduced miR-361-5p and ATMIN and enhanced UBR5 levels show in gliomas cells. A RT-qPCR tested miR-361-5p expression in gliomas cell lines and NHA. B RT-qPCR tested UBR5 mRNA expression in gliomas cell lines and NHA. C, D Western blot tested UBR5 protein expression in gliomas cell lines and NHA. E, F Western blot tested ATMIN protein expression in cells. The data were all measurement data, in the form of mean \pm standard deviation. ${ }^{*} P<0.05$ compared with NHA.

and ATIMN expression levels were reduced in gliomas cell lines while UBR5 level showed the opposite trend (Fig. 2A-F). Thus, U87 and U251 were screened out for follow-up experiments.

\section{Elevating miR-361-5p is anti-tumor for gliomas cells; downregulation of UBR5 reverses the worsening effect caused by miR-361-5p inhibition in gliomas}

The biological effects of miR-361-5p on gliomas cells were explored through gain-of-function and loss-of-function experiments. At the same time, we designed rescue experiment with miR-361-5p inhibitor and UBR5 low expression.

RT-qPCR detected the changes of miR-361-5p expression to ensure success of transfection (Fig. 3A, Supplementary Figure 1A). RT-qPCR and Western bot suggested that MMP-2 level in gliomas cells was suppressed by miR-361-5p mimic while enhanced by miR361-5p inhibitor. Downregulation of UBR5 reversed the elevation of MMP-2 level mediated by miR-361-5p inhibitor (Fig. 3B; Supplementary Figure 1B).

Then, the effects of miR-361-5p on gliomas cell invasion, migration, apoptosis, viability, and colony-forming ability were testified by Transwell assay, RT-qPCR, western blot, flow cytometry, MTT assay, and colony formation assay. As a result of miR-361-5p overexpression, cell migration, invasion, and viability were impaired, colony number was reduced, apoptosis rate was enhanced, and $\mathrm{Bcl}-2$ level was decreased and Bax level was elevated. miR-361-5p downregulation functioned in an opposite way to gliomas cell progression. Silencing UBR5 after interference reversed the effects of miR-361-5p downregulation (Fig. 3C-K; Supplementary Figure 1C-K).

\section{UBR5 is directly targeted by miR-361-5p}

In gliomas cells, UBR5 levels were found to raise after miR-361-5p inhibition. On the contrary, miR-361-5p upregulation suppressed UBR5 levels. Downregulation of UBR5 suppressed the level of UBR5 mediated by miR-361-5p inhibition (Fig. 4A-E). The binding sites were found between UBR5 and miR-361-5p through Starbase website (Fig. 4F), thus miR-361-5p was speculated to target UBR5 in tumorigenesis. For further validation, results from dual-luciferase reporter gene assay demonstrated that cotransfection of miR-361-5p mimic and UBR5-Wt suppressed the luciferase activity of cells (Fig. 4G, H). Finally, we also conducted a correlation analysis and found that miR-361-5p expression was negatively correlated with UBR5 expression in clinical samples (Fig. 4I). 
A $\square$ mimic NC $\square$ inhibitor NC B $\square$ mimic NC $\square$ inhibitor NC $\square$ mimic NC
miR-361-5p mimic $\square$ miR-361-5p inhibitor $\square$ miR-361-5p mimic $\square$ miR-361-5p inhibitor

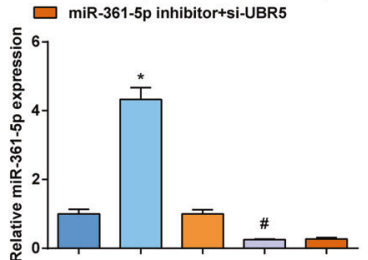

C

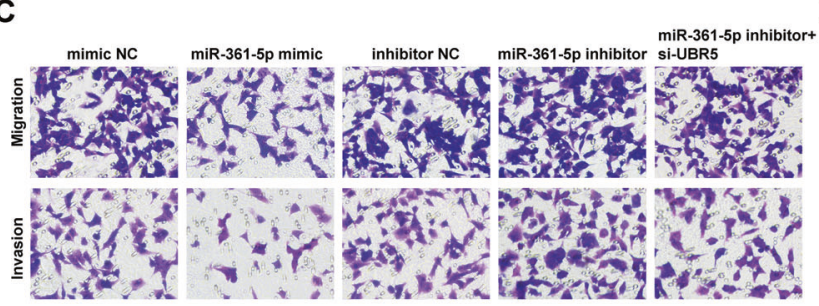

$\mathbf{F}$

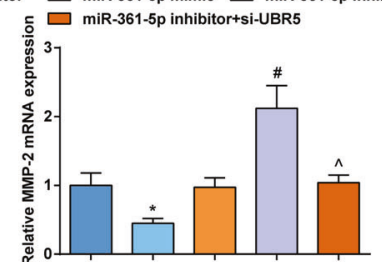

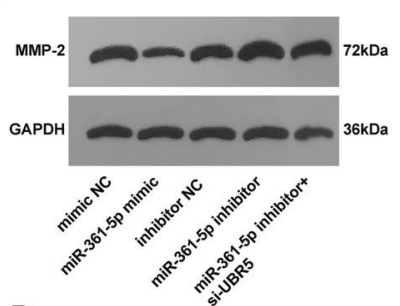

D $\square$ mimic NC $\square$ inhibitor NC

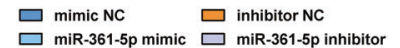

$\square$ miR-361-5p mimic $\square$ miR

miR-361-5p inhibitor+si-UBR

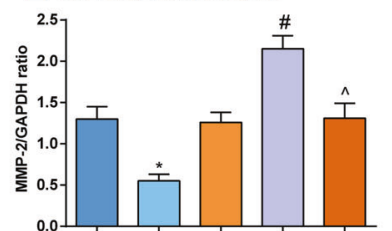

E $\square$ miR-361-5p mimic $\square$ miR-361-5p inhibitor $\square$ miR-361-5p mimic $\square$ miR-361-5p inhibitor $\square$ miR-361-5p inhibitor+si-UBR5

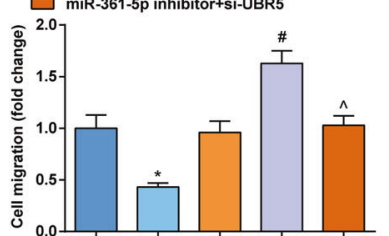

$\square$ miR-361-5p inhibitor+si-UBR5
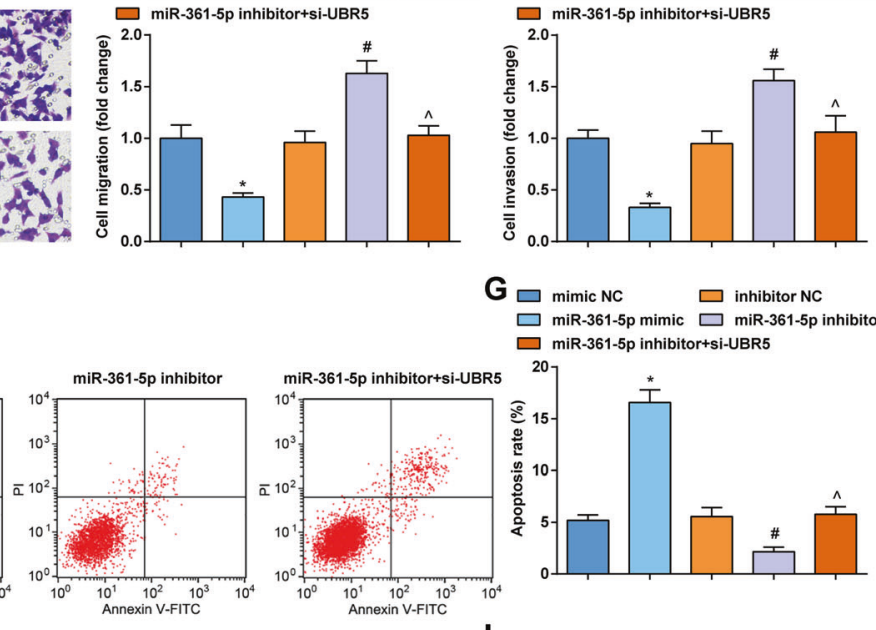

$\mathbf{G} \square$ mimic NC $\square$ inhibitor NC $\square$ miR-361-5p mimic $\square$ miR-361-5p inhibitor miR-361-5p inhibitor+si-UBR5
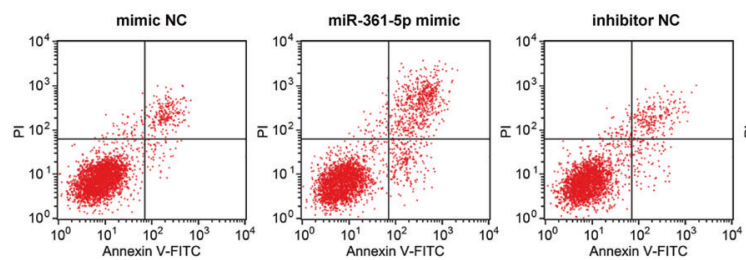

H $\square$ mimic NC $\square$ inhibitor NC

$\square$ miR-361-5p mimic $\square$ miR-361-5p inhibito
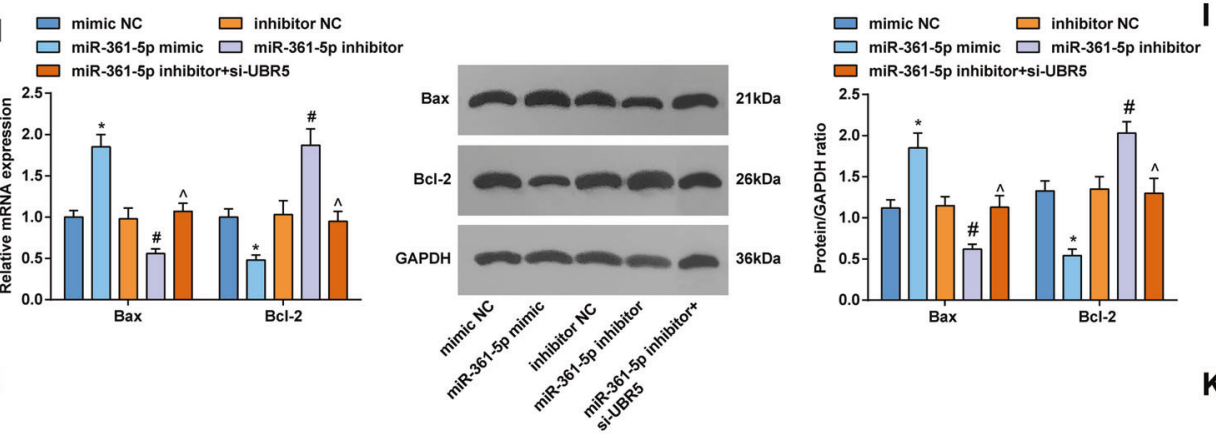

$\square$ miR-361-5p mimic $\square$ miR-361-5 inhibitor+si-UBR5

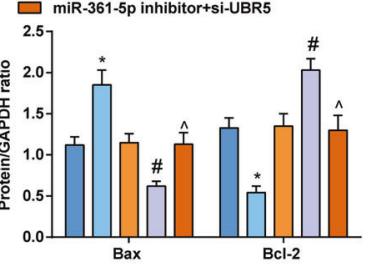

$\rightarrow$ mimic NC $\quad \rightarrow$ inhibitor NC

$\rightarrow$ miR-361-5p mimic $\rightarrow$ miR-361-5p inhibitor

- - miR-361-5p inhibitor +si-UBR5

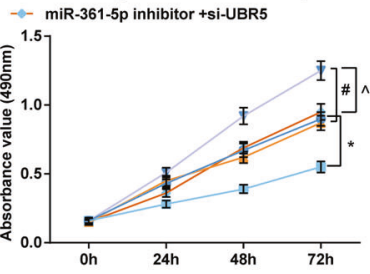

J
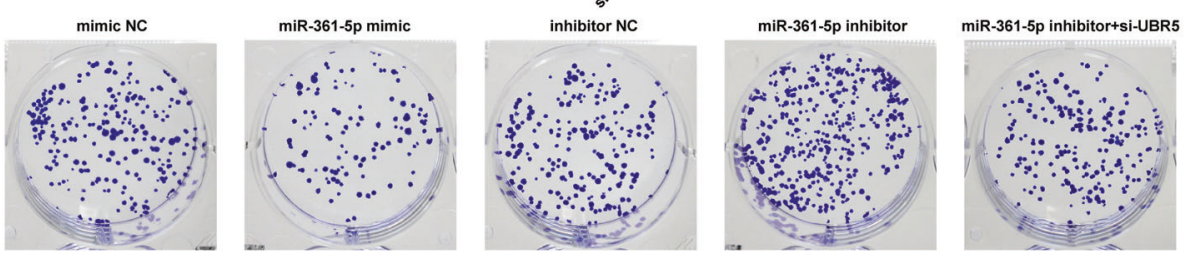

K

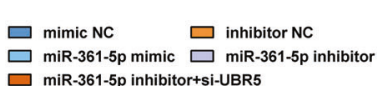

$\square$ miR-361-5p inhibitor+si-UBR5

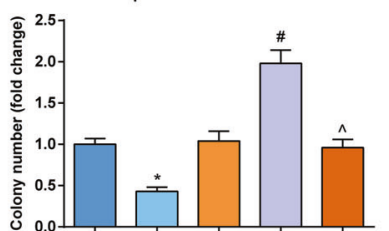

Fig. 3 Elevating miR-361-5p is anti-tumor for gliomas cells; downregulation of UBR5 reverses the worsening effect caused by miR-361-5p inhibition in gliomas. A RT-qPCR tested miR-361-5p expression in U87 cells. B RT-qPCR and western blot tested MMP-2 expression in U87 cells. C-E Transwell tested the invasion and migration of U87 cells. F, G Flow cytometry tested apoptosis of U87 cells. H RT-qPCR and western blot tested Bcl-2 and Bax expression in U87 cells. I MTT tested the viability of U87 cells. J, K Colony formation assay tested the colony-forming ability of U87 cells; the data were all measurement data, in the form of mean \pm standard deviation; ${ }^{*} P<0.05$ compared with the mimic NC group; ${ }^{\#}<0.05$ compared with the inhibitor NC group; $\wedge P<0.05$ compared with the miR-361-5p inhibitor group.

\section{UBR5 regulates ubiquitination of ATMIN}

UBR5 ubiquitinates ATMIN during ionizing radiation to release and promotes ATM activation [13]. Based on that, the effect of UBR5 on gliomas was inferred to relate to ATMIN activity. In the present study, ATMIN protein expression manifested a reduction whereas ATMIN mRNA expression showed no change in gliomas cells after UBR5 downregulation (Fig. 5A-E), implying that UBR5 may regulate ATMIN protein through post-translational modification.

The effect of UBR5 on ubiquitination of ATMIN was determined. The endogenous ATMIN degeneration rate was measured after inhibition of protein synthesis by cycloheximide. In cells, immunoprecipitation observed the ubiquitination between UBR5 and ATMIN and finally demonstrated that knocking out UBR5 would inhibit ATMIN ubiquitination, thud elevating ATMIN expression (Fig. 5F). Moreover, the ubiquitination of ATMIN was related to its degradation, as evident by proteasome inhibitor MG132 maintaining ATMIN protein level.

Western blot detected changes in ATMIN protein expression and finally revealed that overexpression of miR-361-5p promoted while downregulation of miR-361-5p inhibited ATMIN protein 

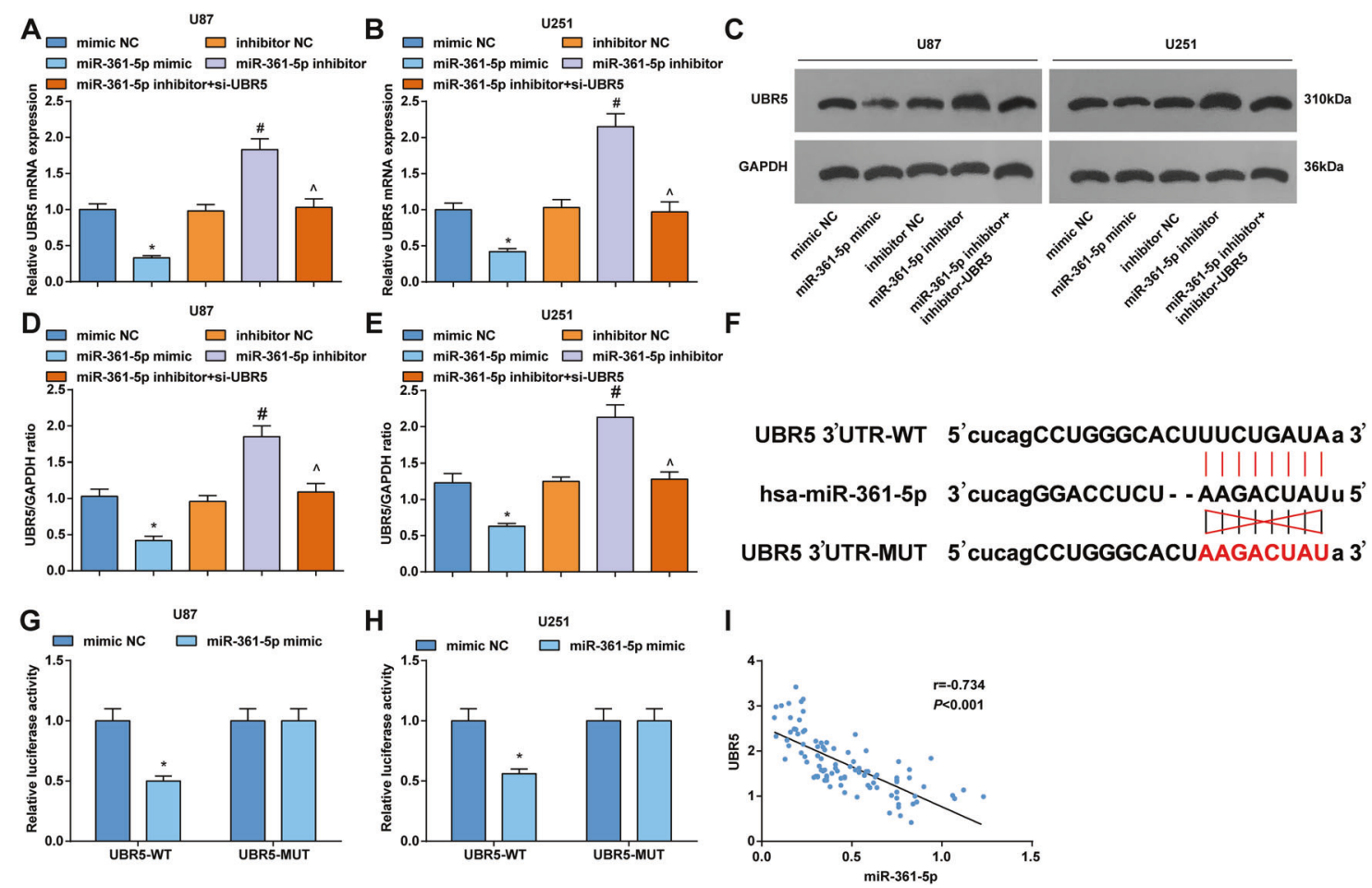

Fig. 4 UBR5 is directly targeted by miR-361-5p. A RT-qPCR tested UBR5 mRNA expression in U87 cells. B RT-qPCR tested UBR5 mRNA expression in U251 cells. C Western blot tested UBR5 protein bands in U87 and U251 cells. D Western blot tested UBR5 protein expression in U87. E Western blot tested UBR5 protein expression in U251 cells. F Starbase predicted the binding sites of miR-361-5p and UBR5. G Dualluciferase reporter gene assay verified the targeting relation of miR-361-5p and UBR5 in U87 cells. $\mathbf{H}$ Dual-luciferase reporter gene assay verified the targeting relation of miR-361-5p and UBR5 in U251 cells. I Correlation analysis between miR-361-5p expression with UBR5 expression in gliomas cells. The data were all measurement data, in the form of mean \pm standard deviation; ${ }^{*} P<0.05$ compared with the mimic NC group; ${ }^{*} P<0.05$ compared with the inhibitor group; $\wedge P<0.05$ compared with the miR-361-5p inhibitor group.

expression. Knockdown of UBR5 reversed the reduction of ATMIN protein expression caused by miR-361-5p downregulation (Fig. 5G, H).

\section{Suppression of UBR5 retards gliomas cell growth}

To verify the role of ATMIN ubiquitination and degradation in gliomas, UBR5 depletion was performed in gliomas cells. As demonstrated, depleting UBR5 reduced MMP-2 level in cells (Fig. $6 \mathrm{~A}$; Supplementary Figure $2 \mathrm{~A}$ ).

After that, the progression of U87 and U251 cells was detected in response to UBR5 depletion-induced ATMIN ubiquitination and degradation. Evidently, UBR5 depletion-induced ATMIN ubiquitination and degradation reduced the acquisition of malignant phenotype of gliomas cells (Fig. 6B-J; Supplementary Figure 2B-J).

\section{Downregulating UBR5 impedes gliomas tumor growth in mice} An in vivo model was established in mice to inspect the regulatory mechanism of UBR5 in gliomas tumor growth. U87 cells delivering downregulated UBR5 were injected into mice and greatly suppressed tumor volume and weight from the 9th day (Fig. 7A-C). RT-qPCR detected the expression of UBR5 in tumor tissues and found that UBR5 expression was reduced in mice treated with si-UBR5 (Fig. 7D). Immunohistochemical staining and TUNEL staining of tumors pictured that UBR5 knockdown decreased Ki67 positive cells and increased cell apoptosis rate (Fig. 7E-G).

\section{DISCUSSION}

Malignant gliomas are most aggressive among adult central nervous system malignancies [29]. In gliomas, much attention has been paid to the relevant mechanisms in cell fate. Our experiments were designed to delve out the role of miR-361-5p in gliomas through regulating UBR5-mediated ubiquitination of ATMIN. miR-361-5p was the suppressed gene in gliomas tissues that was tightly connected with tumor grade. However, ectopic expression of miR-361-5p could limit the acquisition of malignant phenotype of gliomas cells. UBR5 was the target of miR-361-5p that was overexpressed in gliomas, and its silence was anti-tumor for gliomas tumor growth in mice. UBR5, in turn, was the mediator for ubiquitination of ATMIN which delayed gliomas development. To simplify, miR-361-5p hampered gliomas by regulating UBR5 to promote the protein expression of ATMIN (Supplementary Figure 3).

In fact, the truth that the low the miR-361-5p expression, the high the tumor grade has been discovered in gliomas, and increasing miR-361-5p level can limit cellular migration and invasion, as well as abate MMP-2 expression [7]. miR-361-5p presents an inhibited level in gliomas that partially results in the blocked apoptosis and stimulated proliferation [6]. Experimentally measured, miR-361-5p level trends to fall in gliomas and if elevated, miR-361-5p could prevent gliomas cells to migrate and invade [8]. From a novel perspective, miR-361-5p is surveyed to be sponged by COX10 antisense RNA 1, thus driving actin gamma 1-mediated proliferation and straining apoptosis of GBM cells [9]. Commonly proved, miR-361-5p is indeed the tumor suppressor in human cancers, including but not limited to gliomas. In gastric cancer and non-small-cell lung cancer, an inverse link shows between lowly expressed miR$361-5 p$ and advanced tumor stage, and restoring miR-361-5p is devastating to cancer cell aggressive behaviors [30, 31]. In a word, miR-361-5p suppresses gliomas development, just like its anti-tumor effects in other tumors. 

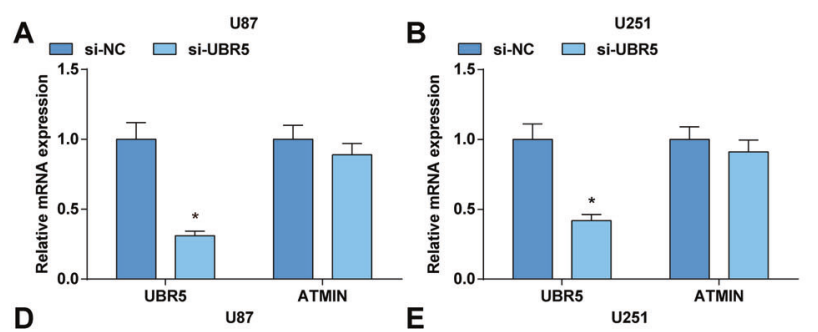

C
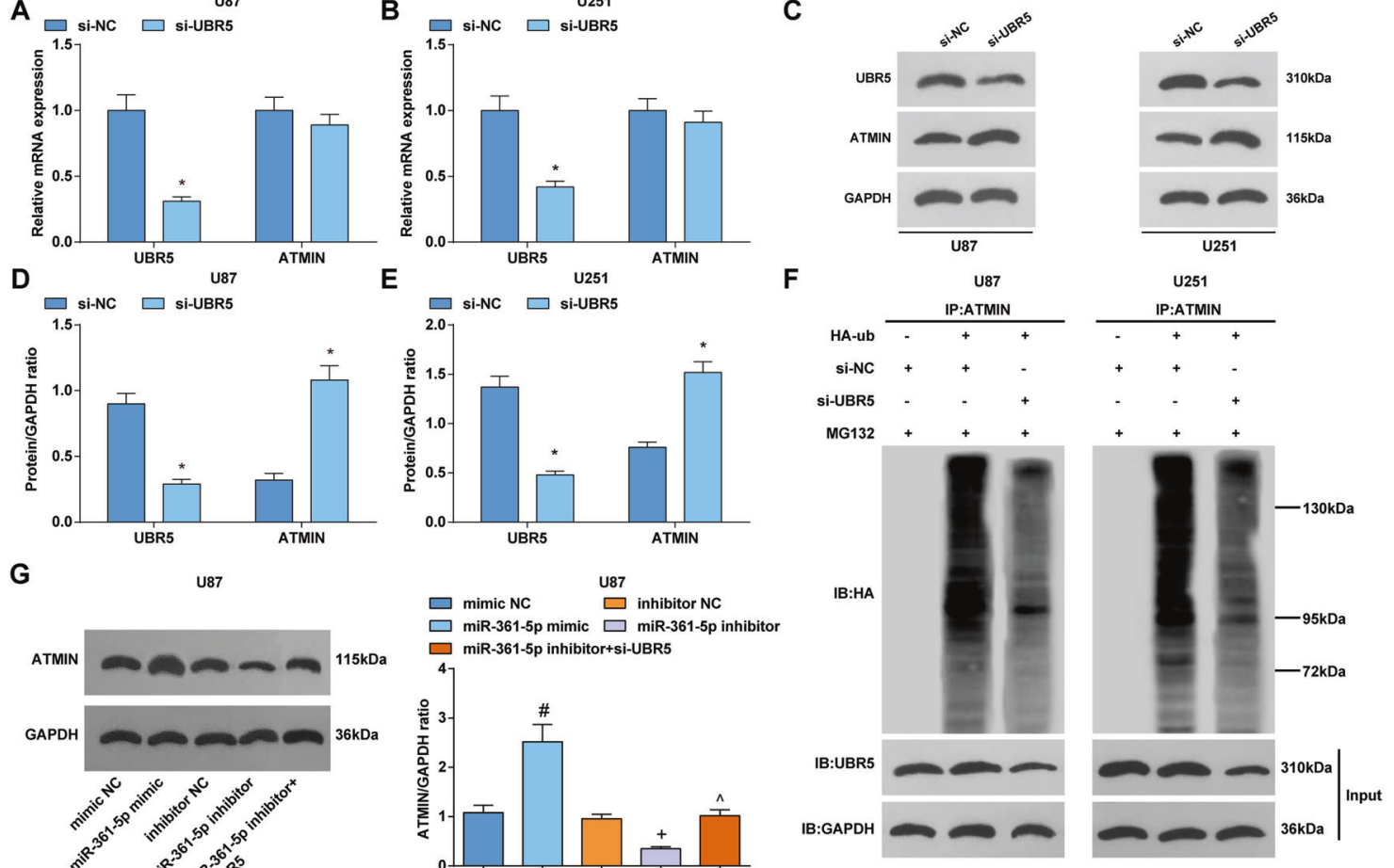

H
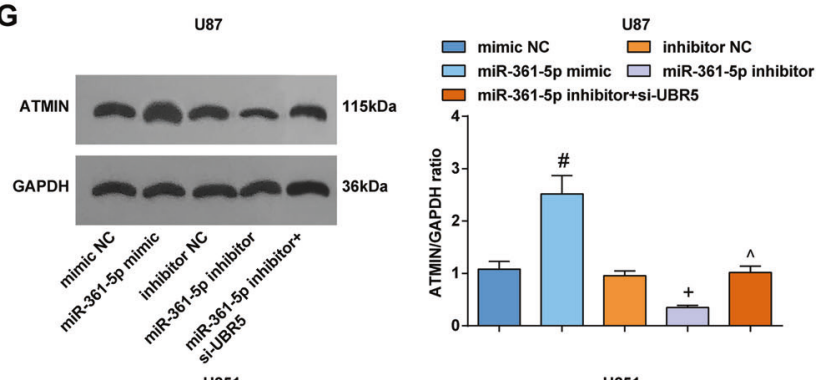

U251
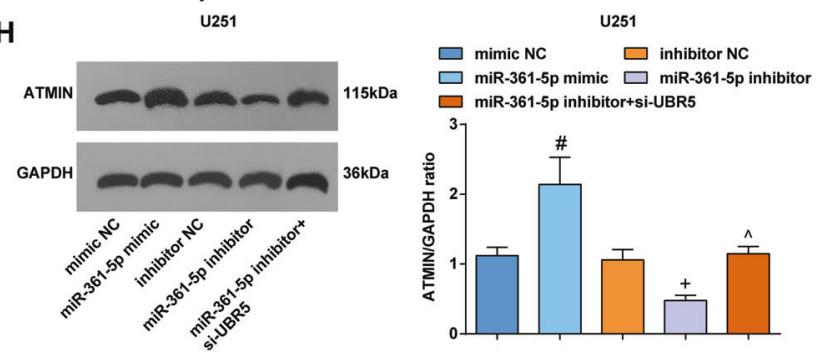

Fig. 5 UBR5 regulates ubiquitination of ATMIN. A RT-qPCR tested UBR5 and ATMIN mRNA expression in U87 cells. B RT-qPCR tested UBR5 and ATMIN mRNA expression in U251 cells. C Western blot tested UBR5 and ATMIN protein bands in U87 and U251 cells. D Western blot tested UBR5 and ATMIN protein expression in U87 cells. E Western blot tested UBR5 and ATMIN protein expression in U251 cells. F Cells were treated with HA-labeled ubiquitin and transfected with siRNA, cells were treated with proteasome inhibitor MG132 (MG) for $2 \mathrm{~h}$ before lysis in denaturing buffer. Immunoprecipitation determined the ubiquitination of ATMIN. G, H Western blot detected ATMIN protein expression in U87 and U251 cells. The data were all measurement data, in the form of mean \pm standard deviation; ${ }^{*} P<0.05$ compared with the si-NC group; ${ }^{\#} P<0.05$ compared with the mimic NC group; ${ }^{+} P<0.05$ compared with the inhibitor NC group; $\wedge P<0.05$ compared with the miR-361-5p inhibitor group.

The targeting relation between UBR5 and miR-361-5p discovered in the present work has not confirmed by previous reports, thereby future investigations are required for verification. UBR5 is enriched in DNA damage repair-related pathway in GBM [32]. In other cancer types, such as gastric cancer, UBR5 level is also enhanced that is associated with advanced TNM stage and depleting UBR5 is the resource for cell proliferation, invasion, and migration inhibition [33]. UBR5 in gallbladder cancer maintains a higher level and repressing UBR5 in cancer cells would remarkably impair cell proliferative and colonyforming capacities [34]. Moreover, the upregulated UBR5 is connected with dismal survival of laryngeal carcinoma patients and the driving player for cell proliferation, invasion, and migration [35]. In addition to that, the pro-tumor role of UBR5 has been convinced in colorectal cancer, judged by its abundant expression in cancer tissues and cells that induces cell proliferation and tumor growth [36]. Widely discovered, UBR5 involves in cancer progression through mediating gene ubiquitination. For instance, UBR5 could promote degradation of sex-determining region $\mathrm{Y}$-box 2, the tumor promoter in esophageal cancer, thereby inhibiting cell proliferation and stemness [11]. In a similar way, UBR5 was examined to promote ubiquitination of ATMIN in gliomas that is consistent with a former study [13]. If UBR5-mediated ubiquitination is suppressed, ATMIN level is tested to elevate [37], further confirming our study results. ATMIN, along with ATM, has been implied as a contributor for GBM formation [15]. Limited studies have revealed the effects of ubiquitination of ATMIN in cancer process, but there are studies mentioning the role of ATMIN in cancers. For example, ATMIN is defined as a tumor inhibitor in lung adenocarcinoma [38].

To summary, the research presents the results clear that miR361-5p modulates the interaction of UBR5 and ATMIN to enhance ATMIN protein expression, thus to repress gliomas development in vitro and in vivo. This research from a novel angle has advanced the potential targets for gliomas treatment. However, the role of miR-361-5p/UBR5/ATMIN axis in gliomas still needs much more explorations and advancements. 
A

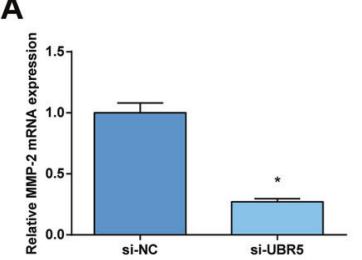

E

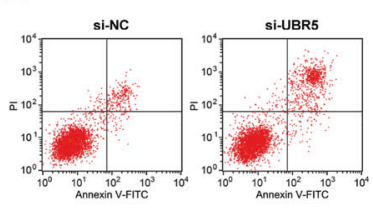

|

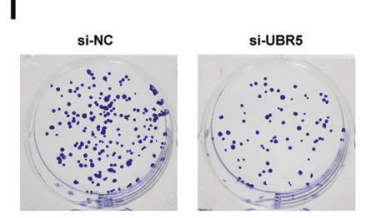

B

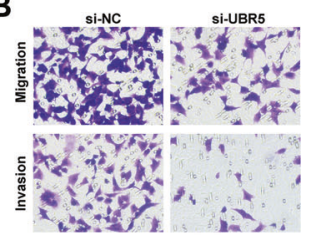

F

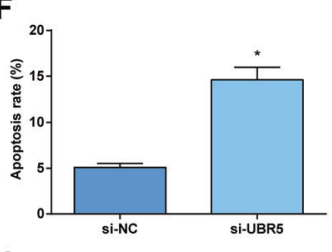

J

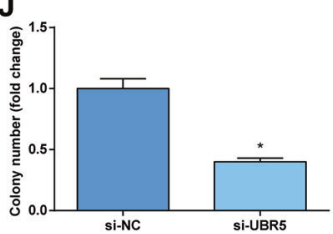

C

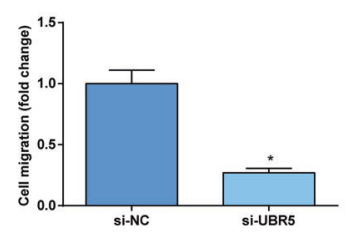

G

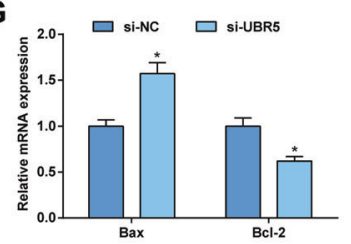

D

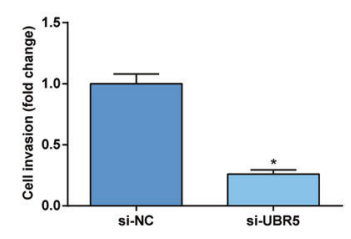

H

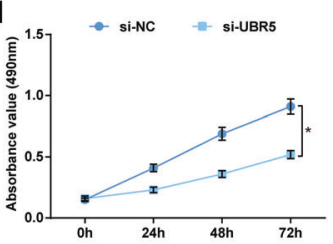

Fig. 6 Suppression of UBR5 retards U87 cell growth. A RT-qPCR tested MMP-2 mRNA expression in U87 cells. B-D Transwell assay tested the migration and invasion of U87 cells. E, F Flow cytometry tested apoptosis of U87 cells. G RT-qPCR tested Bcl-2 and Bax mRNA expression in U87 cells. H MTT tested the optical density of U87 cells at $490 \mathrm{~nm}(0,24,48$, and $72 \mathrm{~h}) . \mathbf{I}$, J Colony formation assay tested the colony-forming ability of U87 cells. The data were all measurement data, in the form of mean \pm standard deviation; ${ }^{*} P<0.05$ compared with the si-NC group.

A

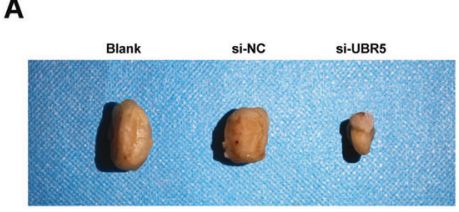

E

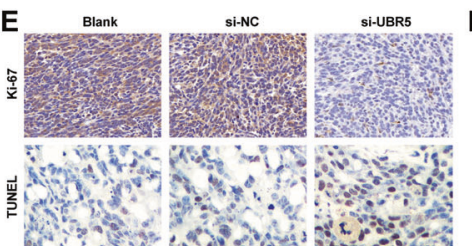

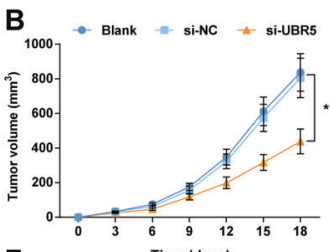

$\mathbf{F}$

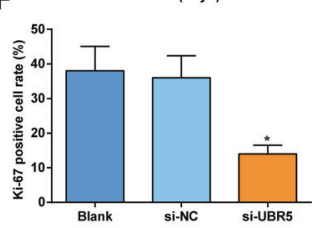

C

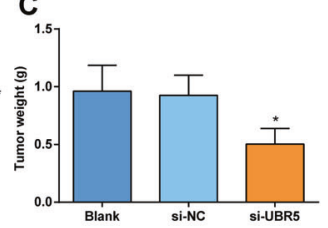

G

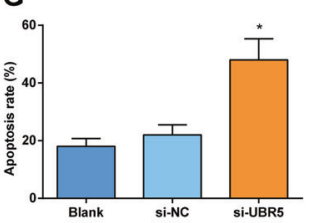

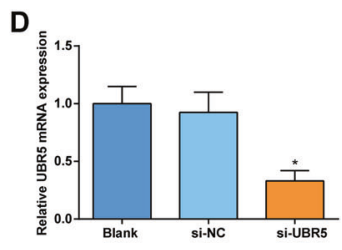

Fig. 7 Downregulating UBR5 impedes gliomas tumor growth in mice. A Tumors of nude mice. B Tumor volume of mice. C Tumor weight of mice. D RT-qPCR detected UBR5 expression in mouse tumors. E Ki-67 immunohistochemical staining results (400x) and TUNEL staining (400x) of tumor tissues of mice. F Ki-67-positive cells in tumor tissues of mice. G Apoptosis rate of cell in tumor tissues of mice; $n=8 / 9$ roup; the data were all measurement data, in the form of mean \pm standard deviation; ${ }^{*} P<0.05$ compared with the si-NC group.

\section{REFERENCES}

1. Perry A, Wesseling P. Histologic classification of gliomas. Handb Clin Neurol. 2016;134:71-95.

2. Louis DN, Perry A, Reifenberger G, von Deimling A, Figarella-Branger D, Cavenee WK, et al. The 2016 World Health Organization Classification of Tumors of the Central Nervous System: a summary. Acta Neuropathologica. 2016;131:803-20.

3. Davis ME. Epidemiology and overview of gliomas. Semin Oncol Nurs. 2018;34:420-9.

4. Xiong L, Wang F, Qi, Xie X. Advanced treatment in high-grade gliomas. J BUON. 2019;24:424-30.

5. Pottoo FH, Javed MN, Rahman JU, Abu-Izneid T, Khan FA. Targeted delivery of miRNA based therapeuticals in the clinical management of Glioblastoma Multiforme. Sem Cancer Biol. 2020;69:391-98.

6. Long N, Chu L, Jia J, Peng S, Gao Y, Yang H, et al. CircPOSTN/miR-361-5p/TPX2 axis regulates cell growth, apoptosis and aerobic glycolysis in glioma cells. Cancer Cell Int. 2020;20:374.

7. Liu J, Yang J, Yu L, Rao C, Wang Q, Sun C, et al. miR-361-5p inhibits glioma migration and invasion by targeting SND1. OncoTargets Ther. 2018;11:5239-52.

8. Zhang X, Wei C, Li J, Liu J, Qu J. MicroRNA-361-5p inhibits epithelial-tomesenchymal transition of glioma cells through targeting Twist1. Oncol Rep. 2017;37:1849-56.
9. Zhou C, Jiang X, Liang A, Zhu R, Yang Y, Zhong L, et al. COX10-AS1 facilitates cell proliferation and inhibits cell apoptosis in glioblastoma cells at post-transcription level. Neurochem Res. 2020;45:2196-203.

10. Jin WL, Mao XY, Qiu GZ. Targeting deubiquitinating enzymes in glioblastoma multiforme: expectations and challenges. Medicinal Res Rev. 2017;37:627-61.

11. Wang $Z$, Kang L, Zhang $H$, Huang $Y$, Fang L, Li M, et al. AKT drives SOX2 overexpression and cancer cell stemness in esophageal cancer by protecting SOX2 from UBR5-mediated degradation. Oncogene 2019;38: 5250-64.

12. Ji SQ, Zhang YX, Yang BH. UBR5 promotes cell proliferation and inhibits apoptosis in colon cancer by destablizing P21. Die Pharmazie. 2017; 72:408-13.

13. Zhang T, Cronshaw J, Kanu N, Snijders AP, Behrens A. UBR5-mediated ubiquitination of ATMIN is required for ionizing radiation-induced ATM signaling and function. Proc Natl Acad Sci USA. 2014;111:12091-6.

14. Li YJ, Lai WT, Chang CC, Kuo MY, Deng YT, Yang CN, et al. Ataxia-telangiectasia mutated interactor regulates head and neck cancer metastasis via KRas expression. Oral Oncol. 2017;66:100-7.

15. Blake SM, Stricker SH, Halavach H, Poetsch AR, Cresswell G, Kelly G, et al. Inactivation of the ATMIN/ATM pathway protects against glioblastoma formation. eLife. 2016; 5: undefined. 
16. Peng L, Ming Y, Zhang L, Zhou J, Xiang W, Zeng S, et al. MicroRNA-30a suppresses self-renewal and tumorigenicity of glioma stem cells by blocking the NT5Edependent Akt signaling pathway. FASEB J. 2020;34:5128-43.

17. Chen W, Hong L, Hou C, Wang Y, Wang F, Zhang J. MicroRNA-585 inhibits human glioma cell proliferation by directly targeting MDM2. Cancer Cell Int. 2020;20:469.

18. Irrera N, D'Ascola A, Pallio G, Bitto A, Mannino F, Arcoraci V, et al. betaCaryophyllene inhibits cell proliferation through a direct modulation of CB2 receptors in glioblastoma cells. Cancers. 2020;12: undefined.

19. Jin S, Li X, Dai Y, Li C, Wang D. NF-kappaB-mediated miR-650 plays oncogenic roles and activates AKT/ERK/NF-kappaB pathways by targeting RERG in glioma cells. Cell Oncol. 2020;43:1035-48.

20. Hay-Koren A, Caspi M, Zilberberg A, Rosin-Arbesfeld R. The EDD E3 ubiquitin ligase ubiquitinates and up-regulates beta-catenin. Mol. Biol. cell. 2011;22:399-411. 1

21. Dai $Y$, Chen Z, Zhao W, Cai G, Wang Z, Wang X, et al. miR-29a-5p regulates the proliferation, invasion, and migration of gliomas by targeting DHRS4. Front Oncol. 2020;10:1772.

22. Liang Z, Wang $X$, Xu X, Xie B, Ji A, Meng S, et al. MicroRNA-608 inhibits proliferation of bladder cancer via AKT/FOXO3a signaling pathway. Mol Cancer. 2017;16:96.

23. Cheng $\mathrm{H}$, Zhao $\mathrm{H}$, Xiao X, Huang $\mathrm{Q}$, Zeng $\mathrm{W}$, Tian $\mathrm{B}$, et al. Long non-coding RNA MALAT1 upregulates ZEB2 expression to promote malignant progression of glioma by attenuating miR-124. Mol Neurobiol. 2020;58:1006-16.

24. Wang $\mathrm{N}$, Zhang $\mathrm{Y}$, Liang $\mathrm{H}$. MicroRNA-598 inhibits cell proliferation and invasion of glioblastoma by directly targeting metastasis associated in colon cancer-1 (MACC1). Oncol Res. 2018;26:1275-83.

25. Wu C, Su J, Long W, Qin C, Wang X, Xiao K, et al. LINC00470 promotes tumour proliferation and invasion, and attenuates chemosensitivity through the LINC00470/miR-134/Myc/ABCC1 axis in glioma. J Cell Mol Med. 2020;24:12094-106.

26. Zhang $X$, Niu W, Mu M, Hu S, Niu C. Long non-coding RNA LPP-AS2 promotes glioma tumorigenesis via miR-7-5p/EGFR/PI3K/AKT/c-MYC feedback loop. J Exp Clin Cancer Res. 2020;39:196.

27. Song L, Liu L, Wu Z, Li Y, Ying Z, Lin C, et al. TGF-beta induces miR-182 to sustain NF-kappaB activation in glioma subsets. J Clin Investig. 2012;122:3563-78.

28. Sun J, Ma Q, Li B, Wang C, Mo L, Zhang X, et al. RPN2 is targeted by miR-181C and mediates glioma progression and temozolomide sensitivity via the wnt/ beta-catenin signaling pathway. Cell Death Dis. 2020;11:890.

29. Montoya ML, Kasahara N, Okada H. Introduction to immunotherapy for brain tumor patients: challenges and future perspectives. Neurooncol Pract. 2020;7:465-76.

30. Tian L, Zhao Z, Xie L, Zhu J. MiR-361-5p inhibits the mobility of gastric cancer cells through suppressing epithelial-mesenchymal transition via the $\mathrm{Wnt} /$ beta-catenin pathway. Gene 2018;675:102-9.

31. Zhuang ZL, Tian FM, Sun CL. Downregulation of miR-361-5p associates with aggressive clinicopathological features and unfavorable prognosis in non-small cell lung cancer. Eur Rev Med Pharmacol Sci. 2016;20:5132-6.

32. Guo J, Yi GZ, Liu Z, Sun X, Yang R, Guo M, et al. Quantitative proteomics analysis reveals nuclear perturbation in human Glioma U87 cells treated with temozolomide. Cell Biochem Funct. 2020;38:185-94.

33. Ding $F$, Zhu X, Song X, Yuan P, Ren L, Chai C, et al. UBR5 oncogene as an indicator of poor prognosis in gastric cancer. Exp Ther Med. 2020;20:7.

34. Zhang Z, Zheng X, Li J, Duan J, Cui L, Yang L, et al. Overexpression of UBR5 promotes tumor growth in gallbladder cancer via PTEN/PI3K/Akt signal pathway. $J$ Cell Biochem. 2019, undefined.

35. Wang K, Tang J, Liu X, Wang Y, Chen W, Zheng R. UBR5 regulates proliferation and radiosensitivity in human laryngeal carcinoma via the p38/MAPK signaling pathway. Oncol Rep. 2020;44:685-97.

36. Wang J, Zhao X, Jin L, Wu G, Yang Y. UBR5 contributes to colorectal cancer progression by destabilizing the tumor suppressor ECRG4. Digestive Dis Sci. 2017;62:2781-9.

37. Li CG, Mahon C, Sweeney NM, Verschueren E, Kantamani V, Li D, et al. PPARgamma interaction with UBR5/ATMIN promotes DNA repair to maintain endothelial homeostasis. Cell Rep. 2019;26:1333-43 e7.
38. Foster H, Ruiz EJ, Moore C, Stamp GWH, Nye EL, Li N, et al. ATMIN is a tumor suppressor gene in lung adenocarcinoma. Cancer Res. 2019;79:5159-66.

\section{ACKNOWLEDGEMENTS}

We would like to acknowledge the reviewers for their helpful comments on this paper.

\section{AUTHOR CONTRIBUTIONS}

$M Z$ and MY finished study design. JJ, ZO, and MW finished experimental studies. $J J, W M$, and ML finished data analysis. JJ finished manuscript editing. All authors read and approved the final manuscript.

\section{FUNDING}

This work was supported by National Natural Science Foundation of China [No. 82001275] and Natural Science Foundation of Hunan Province, China [Grant No: 2019JJ50894].

\section{COMPETING INTERESTS}

The authors declare no competing interests.

\section{ETHICS STATEMENT}

This study was approved and supervised by the animal ethics committee of The Second Xiangya Hospital of Central South University. The treatment of animals in all experiments conforms to the ethical standards of experimental animals.

\section{ADDITIONAL INFORMATION}

Supplementary information The online version contains supplementary material available at https://doi.org/10.1038/s41419-021-04010-1.

Correspondence and requests for materials should be addressed to M.Z. or M.Y.

Reprints and permission information is available at http://www.nature.com/ reprints

Publisher's note Springer Nature remains neutral with regard to jurisdictional claims in published maps and institutional affiliations.

Open Access This article is licensed under a Creative Commons Attribution 4.0 International License, which permits use, sharing, adaptation, distribution and reproduction in any medium or format, as long as you give appropriate credit to the original author(s) and the source, provide a link to the Creative Commons license, and indicate if changes were made. The images or other third party material in this article are included in the article's Creative Commons license, unless indicated otherwise in a credit line to the material. If material is not included in the article's Creative Commons license and your intended use is not permitted by statutory regulation or exceeds the permitted use, you will need to obtain permission directly from the copyright holder. To view a copy of this license, visit http://creativecommons. org/licenses/by/4.0/.

(c) The Author(s) 2021 